\title{
Exposição ao diabetes gestacional: Alterações sobre os parâmetros de estresse oxidativo e acúmulo de ferro esplênico em ratos
}

\author{
Intrauterine Exposure to Maternal Diabetes: Alterations on oxidative stress \\ parameters and deposition splenic iron in rats
}

Recebido em: Aceito em:

$15 / 09 / 2015$

$03 / 11 / 2015$
Eduardo Carvalho LIRA'; Geórgia Maria Ricardo Félix dos SANTOS ${ }^{1}$; José Jairo Teixeira da SILVA ${ }^{1}$; Glória Isolina Boente Pinto DUARTE ${ }^{1}$; Francisco Carlos Almanajás de AGUIAR JÚNIOR ${ }^{2}$; Cristina de Oliveira SILVA²

${ }^{\prime}$ Departamento de Fisiologia e Farmacologia .Centro de Ciências Biológicas. Universidade Federal de Pernambuco. Av. Prof. Moraes Rego, 1235, Cidade Universitária. CEP 50670-901, Recife, Pernambuco, Brasil. ${ }^{2}$ Núcleo de Ciências Biológicas. Centro Acadêmico de Vitória. Universidade Federal de

Pernambuco - CAV/UFPE, Rua Alto do Reservatório, S/N, Bela Vista. CEP 55608-680, Vitória de Santo Antão, PE, Brasil.E-mail: eduardoclira@gmail.com

\section{ABSTRACT}

Recent evidence shows that iron overload is a risk factor for diabetes associates the oxidative stress caused by hyperglycemia. The aim of this work was to investigate the levels of splenic iron as well as the levels of lipid peroxidation and reduced glutathione (GSH) in offspring from diabetic mothers (DG) compared to control mothers (ND). Diabetes mellitus was induced in female Wistar rats by streptozotocin $\left(42 \mathrm{mg} / \mathrm{kg}\right.$, i.p.) injected on the $7^{\text {th }}$ day of pregnancy. Offspring DG and ND were analyzed with six months of age. In these animals, metabolic parameters, such as glucose tolerance was examined. Thiobarbituric acid reactive substances (TBARS) and activities of GSH was performed on the liver tissue. For quantification of the splenic iron, histological sections were analyzed by histomorphometry. Metabolic tests revealed that M-DG offspring also showed impaired glucose tolerance in $15(\mathrm{p}<0,01)$ and $90(\mathrm{p}<0,05)$ minutes. Splenic iron levels were elevated in DG as well as the levels of TBARS in the liver. Hepatic GSH concentration was reduced compared to ND. The results show that glucose intolerance, elevated levels of splenic iron, oxidative stress and reduced glutathione levels in the offspring DG could be associated with the development of diabetes and its complications in adulthood and the that our model of exposure in uterus maternal diabetes induced transient glucose intolerance with major effects on iron metabolism.

Keywords: iron; oxidative stress; gestational diabetes

\section{RESUMO}

Estudos recentes têm associado um aumento do estresse oxidativo desencadeado pela hiperglicemia à sobrecarga de ferro, por mecanismos pouco conhecidos. O objetivo deste estudo foi avaliar os níveis de ferro esplênico, bem como os níveis de peroxidação lipídica e glutationa reduzida (GSH) na prole adulta de ratas com Diabetes gestacional (DG). O DG foi induzido no $7^{\circ}$ dia de gestação em ratas Wistar com dose única de estreptozotocina. Fêmeas controles (ND) receberam veículo. A prole foi dividida em dois grupos: DG e ND. O perfil glicêmico da prole foi analisado pelo teste de tolerância à glicose intraperitoneal (TTGip). O estresse oxidativo foi avaliado através das substâncias reativas ao ácido tiobarbitúrico (TBARS) e níveis de GSH em tecido hepático. Para a quantificação do ferro esplênico, cortes histológicos foram analisados através da coloração de Perls. A prole DG apresentou uma menor tolerância à glicose aos seis meses de idade, o TTGip mostrou aumento da glicemia após $15(\mathrm{p}<0,01)$ e $90(\mathrm{p}<0,05)$ minutos comparado a prole ND. Os níveis de ferro esplênico foram elevados $(\mathrm{p}<0,05)$ na prole DG, bem como os níveis de TBARS no fígado $(\mathrm{p}<0,01)$. A concentração hepática da GSH foi reduzida $(\mathrm{p}<0,01)$ quando comparada ao grupo ND. Os resultados mostram que a intolerância à glicose, os níveis elevados de ferro esplênico e de estresse oxidativo, bem como os níveis reduzidos de glutationa na prole DG podem estar associados ao desenvolvimento do diabetes e suas complicações na vida adulta.

Palavras chave: ferro; estresse oxidativo; diabetes gestacional 
INTRODUÇÃO

O diabetes gestacional (DG) pode ter uma incidência de 1 a 14\%, dependendo da população estudada e dos testes diagnósticos utilizados, contribuindo consideravelmente para as malformações congênitas (1). No Brasil, a prevalência do DG em mulheres com mais de 20 anos, atendidas no Sistema Único de Saúde (SUS), é de $7,6 \%$ (2).

A programação fetal sugere que alterações metabólicas durante períodos críticos do desenvolvimento pré-natal estão diretamente relacionadas a alterações no metabolismo, na estrutura e na função de órgãos e tecidos na vida adulta (3). Dentre elas, a exposição à hiperglicemia durante o período gestacional pode ter um impacto em longo prazo sobre a fisiologia do feto, contribuindo para o desenvolvimento de doenças metabólicas e cardiovasculares na vida adulta (1). Estudos epidemiológicos corroboram esta hipótese ao demonstrarem uma clara relação entre distúrbios no desenvolvimento intrauterino e a susceptibilidade ao desenvolvimento de diabetes mellitus do tipo 2 (DM2) e doenças cardiovasculares (DCV) na vida adulta (4).

Embora não sejam completamente compreendidos os mecanismos envolvidos no desenvolvimento das comorbidades do diabetes mellitus (DM), evidências experimentais e clínicas têm demonstrado que a exposição prolongada à hiperglicemia $\mathrm{e}$ a ação das espécies reativas de oxigênio (EROs) possuem um papel central neste processo $(5,6)$.

É bem compreendido que o estado redox da célula é finamente regulado pelo balanço entre os sistemas oxidantes e antioxidantes, de modo que o desequilíbrio destes gera produção excessiva de radicais livres (7). Em células de mamíferos, a geração de ERO como o ânion superóxido $\left(\mathrm{O}_{2}^{-}\right)$, radical hidroxila $(\mathrm{OH} \bullet)$ e o peróxido de hidrogênio $\left(\mathrm{H}_{2} \mathrm{O}_{2}\right)$ são contrabalanceados por sistemas antioxidantes enzimáticos como a superóxido dismutase (SOD), catalase e glutationa peroxidase (GPX) e não enzimáticos como as vitaminas, glutationa e outros tióis (7), de modo que a manutenção, assim como variações estreitas do estado redox, é fundamental para homeostasia celular.

O estresse oxidativo é um dos mecanismos envolvidos na fisiopatologia de diversas doenças crônicas, inclusive no agravamento do DM $(8,9)$, fato que tem renovado na literatura o interesse no efeito de antioxidantes naturais como alternativa terapêutica para melhorar ou atenuar a hiperglicemia e suas repercussões patológicas sistêmicas.
Usualmente, no DM ocorre o aumento da produção de radicais livres, associado à redução das defesas antioxidantes (10), cujos mecanismos não são completamente compreendidos. É bem demonstrado que a hiperglicemia crônica é determinante neste processo (11).

Diferentes mecanismos têm sido propostos para o aumento na produção de radicais livres no estado hiperglicêmico, dentre eles a metabolização da glicose como a principal fonte de ERO (8); a peroxidação lipídica induzida pela hiperglicemia (12) e a formação do produto Amadori e seus produtos finais de glicosilação avançada (AGE), os quais, por meio de seus receptores celulares, modificam e inativam enzimas (13), estimulam a produção de ERO (14), bem como elevam a atividade transcricional do NF- $\kappa B$, uma via classicamente inflamatória (15). Desta forma, a hiperglicemia crônica, por meio do estresse oxidativo, compromete funcionalmente proteínas celulares, fosfolipídios de membrana (formação do malondialdeído) e ácidos nucléicos, além de estimular a produção de citocinas inflamatórias como o fator de necrose tumoral alfa $(\mathrm{TNF}-\alpha)(8,16)$, eventos fortemente relacionados ao desenvolvimento das comorbidades diabéticas.

Nesta perspectiva, tem-se demonstrado uma associação entre os estoques elevados de ferro, o aumento do estresse oxidativo e o desenvolvimento de algumas doenças crônico-degenerativas como o câncer (17), esteatose hepática (18), Parkinson (19), Alzheimer (20) e DM (21). Uma vez que não existem mecanismos ativos de excreção do ferro, o acúmulo deste íon por fatores hereditários e/ ou por outras condições patogênicas (22) pode gerar alterações importantes no estado redox celular e contribuir para o desenvolvimento do diabetes e suas complicações (23-25).

Evidências experimentais têm sugerido que o excesso de ferro parece influenciar não somente o aparecimento das comorbidades diabéticas, como a resistência à insulina (26) e a retinopatia (27), mas também DCV $(28,29)$. Sabe-se que alterações nos estoques ferro podem repercutir negativamente no DM, ainda que não se compreendam claramente as alterações induzidas pela hiperglicemia durante a gestação no metabolismo deste íon. Além disso, as alterações causadas pelo acúmulo de ferro na vida adulta e suas correlações com o metabolismo de carboidratos em fases críticas do desenvolvimento pré-natal são ainda pouco conhecidas. Neste trabalho, verificamos os efeitos do diabetes gestacional induzido por estreptozotocina (STZ) sobre os níveis de ferro esplênico e o estresse oxidativo na prole adulta de ratas com diabetes gestacional (DG). 
MATERIAIS E MÉTODOS

Animais. Foram utilizados ratos Wistar, provenientes do biotério setorial do Departamento de Fisiologia e Farmacologia da Universidade Federal do Pernambuco, mantidos à temperatura constante de $22 \pm 2^{\circ} \mathrm{C}$, ciclo claro/ escuro padrão (12 horas claro/12 horas escuro), água e ração ad libitum. Os protocolos foram realizados obedecendo as normas preconizadas no "Guide for the Care and Use of Laboratory Animals, publicado pelo "US National Institute of Health" (NIH publicações 85-23, revisado em 1996) e aprovados pelo Comitê de Ética em Experimentação Animal da Universidade Federal de Pernambuco (Processo 23076.037553/2010-10).

Ratas Wistar com 3 meses de idade ( 230 a $250 \mathrm{~g}, \mathrm{n}=7$ por grupo), nulíparas, receberam uma dose $(42 \mathrm{mg} / \mathrm{kg}$ ) única de estreptozotocina (STZ, Sigma ${ }^{\circledR}$, St Louis, MO) no $7^{\circ}$ dia de gestação, via intra peritoneal (i.p.), dissolvida em tampão citrato trissódico $0,01 \mathrm{M}(\mathrm{pH} 4,5)$. Fêmeas-controle receberam apenas o veículo. Ratas com glicemia em jejum acima de $200 \mathrm{mg} / \mathrm{dL}$ foram consideradas diabéticas. Animais com níveis glicêmicos abaixo do valor estipulado foram descartados. Os níveis de glicose foram estimados com glicosímetro $\left(\right.$ Optium mini ${ }^{\circledR}$ ) no $3^{\circ}, 5^{\circ}$ e $14^{\circ}$ dia após a administração de STZ. Após o desmame, as proles foram divididas em dois grupos: controle (ND) e diabetes gestacional (DG).

Teste de tolerância intraperitoneal à glicose (TTGip). As alterações no metabolismo do ferro e do estado redox induzidas pela hiperglicemia foram avaliadas na prole de mães diabéticas aos seis meses de idade. Para confirmar as alterações no metabolismo glicídico nesta fase, foi realizado o TTGip. Após jejum overnight (12h), os animais de ambos os grupos DG e ND foram anestesiados com pentobarbital sódico (50 mg/kg, i.p.) e após 10 minutos da indução anestésica foi administrada uma solução de glicose $50 \%(2 \mathrm{~g} / \mathrm{kg}$, i.p.). Foram coletadas amostras de sangue através de um corte na extremidade da cauda dos animais para determinação da glicemia basal (tempo 0) e após 15 , 30, 60, 90 e 120 minutos após a sobrecarga de glicose, utilizando glicosímetro.

Processamento do material biológico. Amostras de tecido esplênico de ambos os grupos foram mergulhados em uma solução de formol neutra e tamponada $10 \%$, pelo período de 24 horas. Posteriormente, os fragmentos foram desidratados em álcool etílico em concentrações crescentes, diafanizados pelo xilol, impregnados e incluídos em parafina. Para cada fragmento esplênico foram confeccionados cortes semisseriados com espessura de $5 \mu \mathrm{m}$, em seguida fixados em lâminas preparadas com albumina e mantidos em estufa à temperatura de $37^{\circ} \mathrm{C}$, por 24 horas para secagem. Após esse período, os cortes foram submetidos à etapa de desparafinização, coloração e montagem.
Avaliação microscópica e histoquímica. Os cortes foram submetidos à técnica de coloração Hematoxilina-Eosina (H.E.) para a descrição histomorfológica preliminar e por coloração de Perls, para determinar os níveis de deposição de ferro esplênico.

Análise histomorfométrica. Para a avaliação histomorfométrica, as imagens histológicas das lâminas foram capturadas por câmera digital acoplada ao microscópio óptico, sob foco fixo e clareza de campo, obtendo-se 10 campos por lâmina com aumento final de $400 \mathrm{X}$. As fotomicrografias foram avaliadas utilizando o software ImageJ versão 1.44 (Research Services Branch, U.S. National Institutes of Health, Bethesda, MD, USA.); o plugin "color deconvolution", foi utilizado na quantificação dos níveis de ferro tecidual. Os dados obtidos por meio da análise histomorfométrica foram normalizados para o controle (controle $=100 \%)$

Avaliação da peroxidação lipídica A medida dos níveis de peroxidação lipídica foi avaliada pela dosagem das substâncias reativas ao ácido tiobarbitúrico (TBARS), como o malondialdeído (MDA) segundo Ohkawa e cols. (1979) modificado (30). Para cada $1 \mathrm{~g}$ de tecido hepático foram adicionados $5 \mathrm{~mL}$ de KCl-EDTA 1,15\% e homogeneizado por 30 segundos. A seguir, $50 \mu \mathrm{L}$ foram transferidos para um tubo contendo $950 \mu \mathrm{L}$ da solução de ácido tiobarbitúrico (TBA) $0,8 \%$, dodecilsulfato de sódio (SDS) $8,1 \%$, ácido acético $20 \%$ e água, incubando em banho Maria, a $95{ }^{\circ} \mathrm{C}$, por 60 minutos. Posteriormente, os tubos foram imediatamente resfriados em recipiente contendo gelo para bloquear a reação. Foram adicionados $0,25 \mathrm{~mL}$ de água destilada e 1,25 mL de n-butanol, homogeneizando no vórtex por mais 30 segundos. Os tubos foram centrifugados por 10 minutos a 4.000 r.p.m. e a leitura da fase orgânica foi realizada em espectrofotômetro na absorbância de $532 \mathrm{~nm}$. Os resultados foram expressos em $\eta \mathrm{mol} \mathrm{MDA} / \mathrm{mg}$ de proteína.

Avaliação dos níveis de glutationa reduzida. A dosagem da glutationa reduzida (GSH) foi realizada no tecido hepático de acordo com o método de Sedlak e Lindsay (1968) modificado (31). Amostras de tecido foram homogeneizadas em tampão KCl-EDTA 1,15\%. Para cada $400 \mu \mathrm{L}$ de homogenato foram adicionados $400 \mu \mathrm{L}$ de $\mathrm{KCl}$ -EDTA e $400 \mu \mathrm{L}$ de ácido tricloroacético (TCA) 10\%, e centrifugados por 20 minutos a 2400 r.p.m. a $4{ }^{\circ} \mathrm{C}$, para precipitação proteica. Em seguida, $100 \mu \mathrm{L}$ do sobrenadante da amostra foram transferidos para um tubo contendo 400 $\mu \mathrm{L}$ de tampão TRIS-EDTA, $400 \mu \mathrm{L}$ de água destilada e 100 $\mu \mathrm{L}$ de ácido ditionitrobenzóico (DTNB). Após 5 minutos de reação, a leitura foi realizada em espectrofotômetro na absorbância de $412 \mathrm{~nm}$. Os resultados foram expressos em ๆmol GSH/mg de proteína.

O protocolo experimental desenvolvido está apresentado esquematicamente na Figura 1. 
Gestação

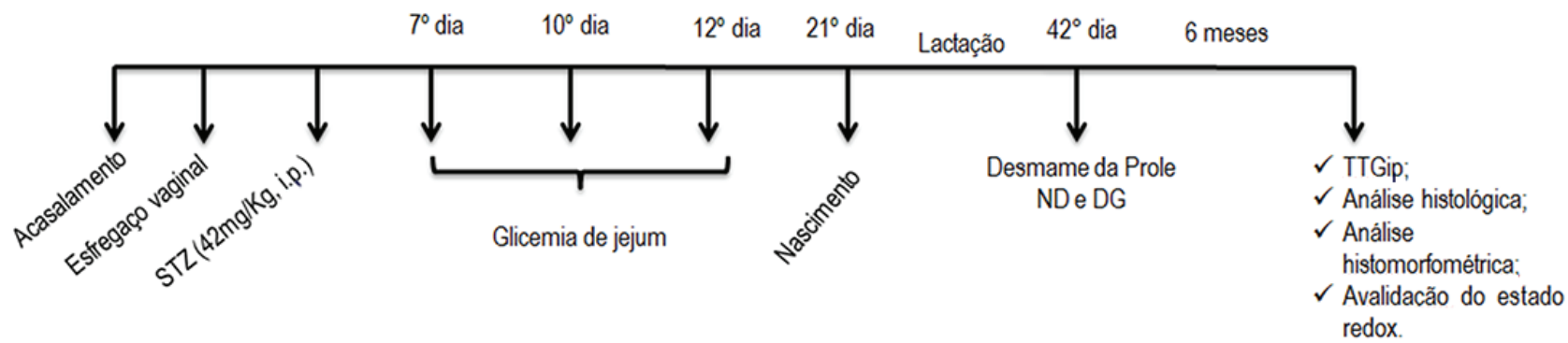

Figura 1: Desenho experimental para avaliação do efeito do diabetes mellitus no estado redox e acúmulo de ferro em prole de rato.

Análise estatística. Os resultados foram expressos em médias \pm erro padrão da média (e.p.m). $\mathrm{O}$ teste $\mathrm{t}$ não -pareado foi utilizado. Para análise de bloco foi utilizada a análise de variância (ANOVA) a duas vias. As diferenças foram consideradas estatisticamente significativas quando $\mathrm{p}<0,05$.

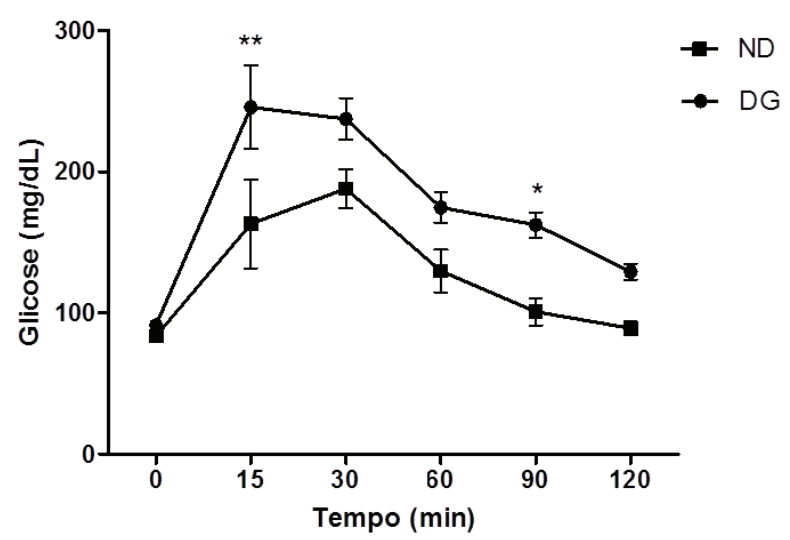

Figura 2: Resultado do teste de tolerância à glicose intraperitoneal (TGip) das proles adultas de animais expostos ao diabetes induzido por estreptozotocina (DG, círculos) no período intrauterino e controles não diabéticos (ND, quadrados). Os valores são expressos como média \pm e.p. $m ; n=5 ;{ }^{*} p<0,05 ;{ }^{* *} p<0,01$ (ANOVA a duas vias).

\section{RESULTADOS E DISCUSSÃO}

Teste de tolerância intraperitoneal à glicose (TTGip). De acordo com a Figura 2, a prole de ratas diabéticas $(\mathrm{DG}, \mathrm{n}=7)$, aos seis meses de idade, apresentou intolerância à glicose após $15(\mathrm{p}<0,01)$ e $90(\mathrm{p}<0,05)$ minutos em comparação com a prole ND $(n=7)$. Área sob a curva (ND $16000 \pm 810$ vs. DG $22000 \pm 920, \mathrm{n}=5, \mathrm{p}<0,001$ ) (Figura 3).

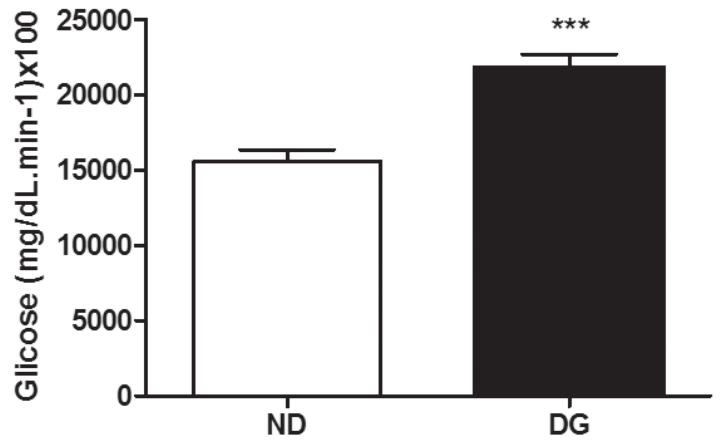

Figura 3: Área sob a curva das proles de animais expostos ao diabetes induzido por estreptozotocina (DG, barras pretas) no período intra-uterino e controles não diabéticos (ND, barras brancas). 0 s valores são expressos como média \pm e.p.m; $n=5 ;{ }^{*} p<0,001$ (ANOVA a duas vias).

Avaliação do ferro esplênico utilizando histomorfometria. Aos seis meses de idade, a análise histológica do baço do grupo DG apresentou um aumento $(\mathrm{p}<0,05)$ nos níveis de ferro em comparação a ND $(120 \pm 4,2$ vs. $100 \pm$ 4,7 , respectivamente, $n=7$, Figura 4; Tabela 1). Os níveis de ferro esplênico também foram elevados nas mães submetidas ao diabetes gestacional induzido por STZ (M-DG), em comparação as mães controles (M-ND) $(120,0 \pm 3,1$ vs. $100,0 \pm 3,0$, respectivamente, Figura 5).

Tabela 1: Análise histomorfométrica quantitativa de ferro esplênico da prole adulta de animais expostos ao diabetes induzido por estreptozotocina ( $42 \mathrm{mg} / \mathrm{kg}$ i.p.) no período intra-uterino (DG) e controles não diabéticos (ND). 
Quantificação de TBARS e GSH teciduais. Os níveis de TBARS no fígado foram significativamente elevados $(\mathrm{p}<0,01)$ no grupo DG $(0,40 \pm 0,019, \mathrm{n}=7)$ quando comparado a ND $(0,28 \pm 0,020, \mathrm{n}=7$, Figura 6). Em relação à concentração hepática da GSH, o grupo DG apresentou uma redução $(\mathrm{p}<0,01)$ nos níveis dessa enzima em comparação a prole oriunda de mães controles (DG [80 \pm $2,8, \mathrm{n}=7]$ vs. ND [96 $\pm 3,8, \mathrm{n}=4]$, Figura 7).

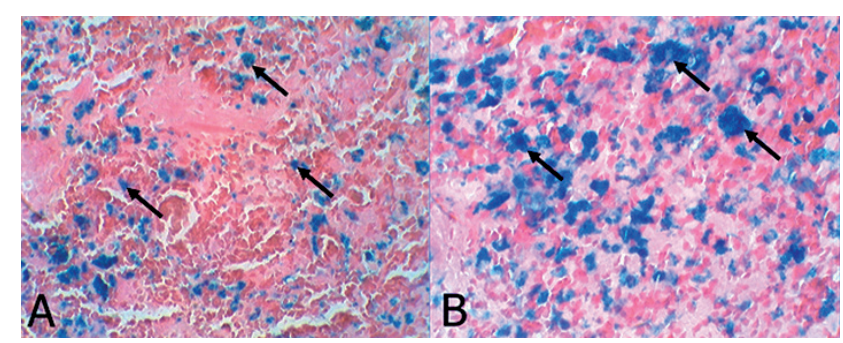

Figura 4: Fotomicrografias de cortes histológicos do baço. Aumento final de $400 x$, coloração de Perls. "A" prole adulta de animais controles não diabéticos (ND) e "B" animais expostos ao diabetes induzido por estreptozotocina (42 $\mathrm{mg} / \mathrm{kg}$ i.p.) no período intra-uterino (DG). Notar presença de depósitos de ferro em azul indicado pelas setas.

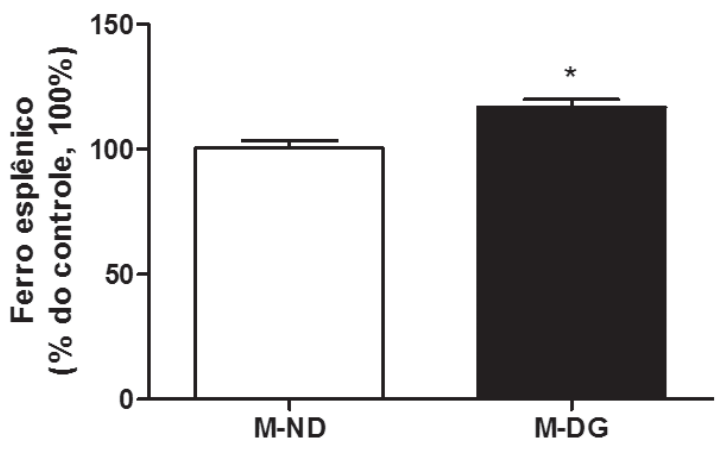

Figura 5: Área ocupada pelos depósitos de ferro no baço de fêmeas (mães) com diabetes gestacional (M-DG, barras pretas) induzido por estreptozotocina (42 mg/kg, i.p.) e fêmeas não diabéticas (M-ND, barras brancas). 0 eixo $Y$ mostra a percentagem de valores de coloração relativos, em comparação com os animais controles $(100 \%)$. Os valores são expressos como média \pm e.p.m; ${ }^{*} p<0,05$ (Teste t de Student não pareado)

Quantificação de TBARS e GSH teciduais. Os níveis de TBARS no fígado foram significativamente elevados $(\mathrm{p}<0,01)$ no grupo DG $(0,40 \pm 0,019, \mathrm{n}=7)$ quando comparado a ND $(0,28 \pm 0,020, \mathrm{n}=7$, Figura 6). Em relação à concentração hepática da GSH, o grupo DG apresentou uma redução $(p<0,01)$ nos níveis dessa enzima em comparação a prole oriunda de mães controles (DG [80 \pm $2,8, \mathrm{n}=7]$ vs. ND [96 $\pm 3,8, \mathrm{n}=4]$, Figura 7).

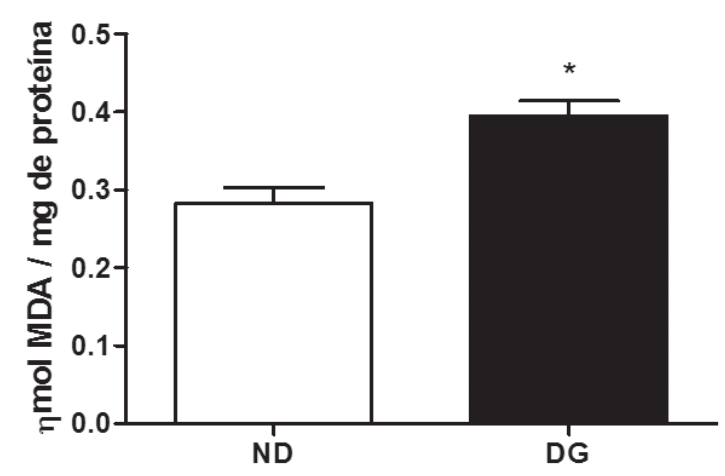

Figura 6: Comparação entre os níveis de substâncias reativas ao ácido tiobarbitúrico (TBARS) das proles adultas de animais expostos ao diabetes induzido por estreptozotocina (DG, barras pretas) no período intra-uterino e controles não diabéticos (ND, barras brancas). Os valores são expressos como média \pm e.p.m; * $p<0,05$ (Teste t de Student não pareado).

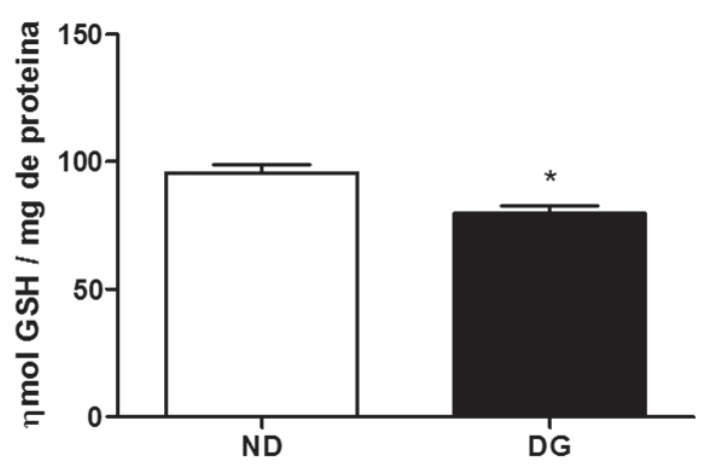

Figura 7: Comparação entre os níveis de glutationa reduzida (GSH) das proles adultas de animais expostos ao diabetes induzido por estreptozotocina (DG, barras pretas) no período intra-uterino e controles não diabéticos (ND, barras brancas). Os valores são expressos como média \pm e.p.m; ${ }^{*} p<0,05$ (Teste $t$ de Student não pareado).

Estudos experimentais e clínicos têm demonstrado que hiperglicemia, durante o período gestacional, modifica a homeostase fetal, o que está relacionado a diferentes complicações neonatais como a macrossomia, hipoglicemia, hipocalcemia e a hiperbilirrubinemia, além de uma maior predisposição a obesidade, DM2 e hipertensão arterial na vida adulta $(32,33)$.

Neste estudo, os animais oriundos de mães diabéticas não apresentaram diferença significativa quanto ao peso corporal em comparação à prole de ratas controles aos seis meses de idade $(500 \pm 0,62$ vs. $490 \pm 8,1$, respectivamente, $\mathrm{n}=7$, Figura 8). 
As consequências no peso corporal do feto ou neonatos de ratas diabéticas dependem não somente do nível de glicemia durante a gestação, mas também do período de vida estudado. Em roedores foi demonstrado que uma exposição intrauterina a baixas doses de STZ durante a gestação está associada com peso normal ou macrossomia $(34,35)$. Por outro lado, estudos realizados com a prole de mães submetidas a altas doses de STZ evidenciaram que os animais apresentaram redução do crescimento na idade adulta $(36,37)$.

A hiperglicemia per se gera AGE que induzem a formação das ERO na presença de metais como ferro. Além disso, metais de transição como o ferro, extremamente abundante na placenta, estão diretamente relacionados ao estresse oxidativo (38). Neste sentido, o ferro potencializa os danos causado por agentes oxidantes (39), o que o torna um fator de risco para o desenvolvimento do diabetes e suas complicações, por promover o aumento da produção de ERO (40). Estudos mostram que alterações nos níveis de ferro corporal podem estar presentes em adultos com DM2 (41-43) na obesidade (44), síndrome metabólica (45) e DG (46).

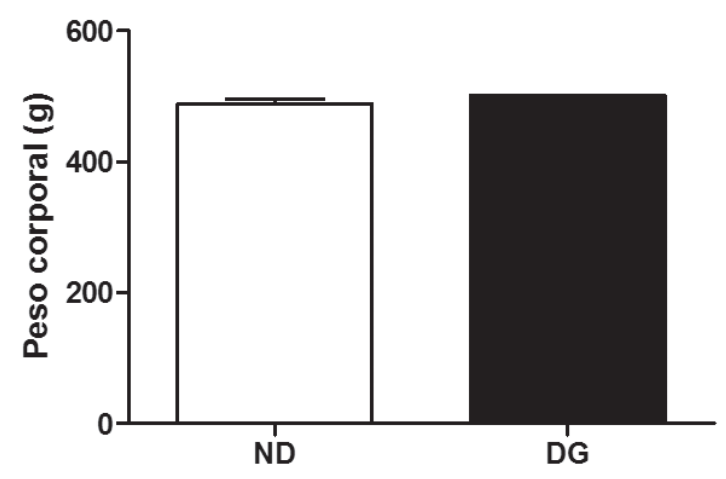

Figura 8: Massa corporal aos seis meses de idade das proles de animais expostos ao diabetes induzido por estreptozotocina (DG, barras pretas) no período intra-uterino e controles não diabéticos (ND, barras brancas). Os valores são expressos como média \pm e.p.m; $n=8 ; p>0,05$ (Teste t de Student não pareado).

Dados epidemiológicos também mostram a relação entre estoques de ferro corporal e estados pré-diabéticos nos quais há alterações de níveis glicêmicos no jejum e na tolerância à glicose (47) o que corrobora com os resultados encontrados na prole de ratas com DG que apresentaram intolerância a glicose. Ademais, nossos dados também são compatíveis com a hipótese de que a exposição intrauterina à níveis elevados de glicose constitui um fator de risco para a prole no desenvolvimento da intolerância à glicose, bem como, de outras alterações metabólicas na vida adulta.

No presente trabalho, foram observadas concentrações mais elevadas de ferro esplênico na prole de ratas DG. Acredita-se que exista uma vulnerabilidade diferente de cada tecido à interação do ferro no diabetes (48). É impor- tante ressaltar que o ferro corporal é armazenado predominantemente no sistema retículo-endotelial, principalmente nas células reticulares do baço e da medula óssea onde fica armazenado na forma de ferritina e hemossiderina (49). O aumento dos níveis de ferro excede a capacidade de ligação à transferrina, o que resulta no acúmulo de ferro pelos hepatócitos, cardiomiócitos e células acinares pancreáticas. $\mathrm{O}$ ferro livre, não ligado a transferrina, tem forte tendência de mediar reações que levam à produção de ERO provocando danos celulares como a peroxidação lipídica (50-52). Uma das possíveis explicações para as alterações no metabolismo do ferro encontradas no diabetes seria a redistribuição dos receptores de transferrina do compartimento intracelular para a membrana plasmática pela insulina, o que levaria a um aumento da síntese de ferritina e consequentemente aumento do consumo do ferro nos tecidos $(48,53,54)$.

Resultados obtidos em modelo de prole diabética são parcos; alguns estudos realizados em ratos diabéticos submetidos a uma suplementação de ferro apresentaram menores concentrações de ferro no soro (48). Neste modelo de diabetes induzido por STZ, sabe-se que há destruição das células beta pancreáticas, promovendo deficiência de insulina que poderia causar uma maior deposição de ferro $(48,55)$. É importante ressaltar que animais oriundos de fêmeas diabéticas com seis meses de idade apresentam intolerância à glicose e resistência à insulina (56). Em outro estudo, a restrição materna de ferro reduziu os níveis de triglicerídeos na prole com três meses de idade, demonstrando que o ferro pode ter efeitos na fisiologia e metabolismo da prole (57).

A expressão de hepcidina pode estar alterada em pacientes diabéticos (58), podendo representar uma das principais causas para a sobrecarga de ferro (59). Além da hepcidina, a ferritina também possui um importante papel no metabolismo do ferro. Ela está presente principalmente nas células do sistema retículo endotelial do fígado, baço e medula óssea sendo responsável pelo armazenamento e conversão intracelular do ferro bivalente em ferro trivalente para que ele seja utilizado nos diversos processos celulares (60). Entretanto devido a altas concentrações de ferro, a capacidade de mobilização desse metal pela ferritina é saturada. Por isso, as micelas férricas são incorporadas por lisossomos formando agregados que podem ser visualizados, utilizando microscopia óptica, na forma de grânulos, conhecidos como grânulos de hemossiderina. Na reação com o Azul da Prússia (ou Perls), o ferro em sua forma trivalente reage com o ferrocianeto de potássio originando o ferrocianeto férrico, de cor azul. (61), o que foi observado em nossos resultados.

Uma das formas de avaliar o status oxidativo celular é analisar os produtos gerados durante os processos oxidativos (62). Durante a peroxidação lipídica ou lipoperoxidação, por exemplo, há aumento na produção de substâncias reativas ao ácido tiobarbitúrico entre elas o malondialdeído (MDA), resultante da ação de ERO sobre os lipídeos insaturados das membranas celulares, o que leva a alterações 
dos mecanismos de seletividade/ transporte, modificações no DNA, comprometimento de sua estrutura e dos componentes da matriz extracelular, e até mesmo morte celular (39). Portanto a concentração de MDA pelo método das substâncias reativas ao ácido tiobarbitúrico (TBARS) é utilizada para estimar a intensidade da peroxidação lipídica em células e tecidos (63). Nossos resultados mostram um aumento na produção de TBARS na prole submetida ao DG o que pode ser considerado uma evidência da produção de ERO como resultado da diminuição da atividade dos sistemas de defesa antioxidante. Outros estudos em modelos animais também demonstraram que o excesso de ferro pode aumentar os níveis de TBARS no fígado $(64,65)$.

A concentração de grupos tióis, assim como os níveis de TBARS, também pode ser considerada um biomarcador do estresse oxidativo, visto que esses grupos contribuem significativamente na defesa antioxidante celular. A glutationa (GSH) em sua forma reduzida, representa o grupo tiol não proteico mais predominante no organismo, a qual atua como substrato enzimático para outros agentes antioxidantes e participa da síntese de proteínas e DNA $(66,67)$. A GSH também participa da proteção a lesões causadas por metais como o íon ferro (68) e da eliminação de produtos gerados na lipoperoxidação (69). Assim, a depleção de GSH pode prejudicar a atividade de outras enzimas antioxidantes e o dano oxidativo resultante pode então contribuir para a patogênese do diabetes e suas complicações. Os resultados obtidos no presente estudo demonstram uma redução nos níveis de GSH, que em conjunto indicam um aumento do estresse oxidativo na prole DG.

\section{CONCLUSÃO}

Em resumo, a prole adulta oriunda de fêmeas com diabetes gestacional induzido por STZ, apresentou intolerância à glicose, aumento nos níveis esplênicos de ferro e estresse oxidativo, bem como redução nos níveis de GSH no fígado. Os achados deste estudo mostram que existe uma susceptibilidade do baço para o acúmulo de ferro, a qual deve ser investigada, pois poderá promover injúria celular e agravamento do diabetes.

\section{AGRADECIMENTOS:}

Aos órgãos de fomento à pesquisa: Coordenação de Aperfeiçoamento de Pessoal de Nível Superior (CAPES), Conselho Nacional de Desenvolvimento Científico e Tecnológico (CNPq) e Fundação de Amparo à Ciência e Tecnologia do Estado de Pernambuco (FACEPE), pelo suporte financeiro.

\section{REFERÊNCIAS}

1. ADA. American Diabetes Association. Diagnosis and classification of diabetes mellitus. Diabetes Care. 2010. 33(Suppl 1):S62-S69. DOI: 10.2337/dc10-S062.

2. BRASIL. Ministério da Saúde. Gestação de alto risco: manual técnico. $5^{\mathrm{a}}$ ed. Brasília; 2010.

3. Barker DJ. The origins of the developmental origins theory. J Intern Med. 2007. 261(5):412-7. DOI: 10.1111/j. 1365-2796.2007.01809.x.

4. Pinney SE, Simmons RA. Metabolic programming, epigenetics, and gestational diabetes mellitus, Curr Diab Rep. 2012. 12(1):67-74. DOI: 10.1007/s11892-011-02481.

5. Klein R. Hyperglycemia and microvascular and macrovascular disease in diabetes. Diabetes Care. 1995.18(2):25868.

6. Giacco F, Brownlee M. Oxidative stress and diabetic complications. Circ Res. 2010. 107(9): 1058-70. DOI: 10.1161/CIRCRESAHA.110.223545.

7. Bonetto AA, Penna F, Muscaritoli M, Minero VG, Fanelli FR, Baccino FM, Costelli P. Are antioxidants useful for treating skeletal muscle atrophy? Free Radic Biol Med. 2009. 47(7):906-16. DOI: 10.1016/j.freeradbiomed.2009.07.002.

8. Maritim AC, Sanders RA, Watkins JB. Diabetes, oxidative stress, and antioxidants: a review. J Biochem Mol Toxicol. 2003. 17(1):24-38. DOI: 10.1002/jbt.10058
9. Ceriello A. Oxidative stress and glycemic regulation. Metabolism. 2000. 49(2 Suppl 1):27-9.

10. Sharma M, Akhtar N, Sambhav K, Shete G, Bansal AK, Sharma SS. Emerging Potential of Citrus Flavanones as an Antioxidant in Diabetes and its Complications. Curr Top Med Chem. 2014.

11. Banerjee M, Vats P. Reactive metabolites and antioxidant gene polymorphisms in Type 2 diabetes mellitus. Indian J Hum Genet. 2014. 20(1):10-9. DOI: 10.4103/09716866.132747.

12. Kawamura M, Heinecke JW, Chait A. Pathophysiological concentrations of glucose promote oxidative modification of low density lipoprotein by a superoxide-dependent pathway. J Clin Invest. 1994. 94(2):771-8. DOI: 10.1172/ JCI117396.

13. Nédic O, Rattan SIS, Grune T, Trougakos IP. Molecular effects of advanced glycation end products on cell signalling pathways, ageing and pathophysiology. Free Radic Res. 2013. 47(Suppl 1):28-38. DOI: 10.3109/10715762.2013.806798.

14. Baynes JW, Thorpe SR. Role of oxidative stress in diabetic complications: A new perspective on an old paradigm. Diabetes. 1999. 48(1):1-9. DOI: 10.2337/diabetes.48.1.1.

15. Mohamed AK, Bierhaus A, Schiekofer S, Tritschler H, Ziegler R, Nawroth PP. The role of oxidative stress and NF-kappaB activation in late diabetic complications. Bio- 
factors. 1999. 10(2-3):157-67.

16. Singh R, Kaur N, Kishore L, Kumar GG. Management of diabetic complications: A chemical constituents based approach. J Ethnopharmacol. 2013. 150(1):51-70. DOI: 10.1016/j.jep.2013.08.051.

17. Akatsuka $\mathrm{S}$ et al. Fenton reaction induced cancer in wild type rats recapitulates genomic alterations observed in human cancer. PLoS One. 2012. 7(8):e43403. DOI: 10.1371/journal.pone.0043403.

18. Sikorska K, Stalke P, Romanowski T, Rzepko R, Bielawski KP. Liver steatosis correlates with iron overload but not with HFE gene mutations in chronic hepatitis C. Hepatobiliary Pancreat Dis Int. 2013. 12(4):377-84. DOI: 10.1016/S1499-3872(13)60059-4

19. Zhang J, Zhang Y, Wang J, Cai P, Luo C, Qian Z, Dai Y, Feng H. Characterizing iron deposition in Parkinson's disease using susceptibility-weighted imaging: an in vivo MR study. Brain Res. 2010. 1330:124-30. DOI: 10.1016/j. brainres.2010.03.036.

20. Zhu WZ, Zhong WD, Wang W, Zhan CJ, Wang CY, Qi JP, Wang JZ, Lei T. Quantitative MR phase-corrected imaging to investigate increased brain iron deposition of patients with Alzheimer disease. Radiology. 2009. 253(2):497-504. DOI: 10.1148/radiol.2532082324.

21. Huang J, Jones D, Luo B, Sanderson M, Soto J, Abel ED, Cooksey RC, McClain DA. Iron overload and diabetes risk: a shift from glucose to Fatty Acid oxidation and increased hepatic glucose production in a mouse model of hereditary hemochromatosis. Diabetes. 2011. 60(1):80-7. DOI: $10.2337 / \mathrm{db} 10-0593$.

22. Emerit J, Beaumont C, Trivin F. Iron metabolism, free radicals, and oxidative injury. Biomed Pharmacother. 2001. 55(6):333-9. DOI:10.1016/S0753-3322(01)00068-3.

23. Wilson JG, Lindquist JH, Grambow SC, Crook ED, Maher JF. Potential role of increased iron stores in diabetes. Am. J. Med. Sci. 2003. 325(6):332-9.

24. Jomova K, Valko M. Advances in metal-induced oxidative stress and human disease. Toxicology. 2011. 283(23):65-87. DOI: 10.1016/j.tox.2011.03.001.

25. Fernández-Real JM, López-Bermejo A, Ricart W. Cross-talk between iron metabolism and diabetes. Diabetes. 2002. 51(8):2348-54. DOI:10.2337/diabetes.51.8.2348

26. Wlazlo N, Van Greevenbroek MM, Ferreira I, Jansen EH, Feskens EJ, van der Kallen CJ, Schalkwijk CG, Bravenboer B, Stehouwer CD. Iron metabolism is associated with adipocyte insulin resistance and plasma adiponectin: the Cohort on Diabetes and Atherosclerosis Maastricht (CODAM) study. Diabetes Care. 2013. 36(2):309-15. DOI: $10.2337 / \mathrm{dc} 12-0505$.

27. Ciudin A, Hernández C, Simó R. Iron overload in diabetic retinopathy: a cause or a consequence of impaired mechanisms? Exp Diabetes Res. 2010. 2010:1-8. DOI: 10.1155/2010/714108.

28. Burt MJ, Halliday JW, Powell LW. Iron and coronary heart disesase. BMJ. 1993. 307(6904): 575-576.

29. Eftekhari MH, Mozaffari-Khosravi H, Shidfar F. Zamani A. Relation between Body Iron Status and Cardiovascular Risk Factors in Patients with Cardiovascular Disease. Int J Prev Med. 2013. 4(8):911-6.

30. Ohkawa H, Ohishi N, Yagi K. Assay for Lipid Peroxides in animal tissues by thiobarbituric acid reaction. Anal Biochem. 1979. 95(2):351-8. PMID: 36810.

31. Sedlak J, Lindsay RH. Estimation of total, protein-bound, and nonprotein sulfhydryl groups in tissue with Ellman's reagent. Anal Biochem. 1968. 25(1):192-205. PMID: 4973948.

32. Holemans K, Gerber R, Meurrens K, De Clerck F, Poston L, Van Assche FA. Streptozotocin diabetes in the pregnant rat induces cardiovascular dysfunctions in adult offspring. Diabetologia. 1999. 42(1):81-89.

33. Simeoni U, Barker DJ. Offspring of diabetic pregnancy: Long-term outcomes. Seminars in Fetal \& Neonatal Medicine. 2009. 14(2):119-124. DOI:10.1016/j. siny.2009.01.002.

34. Mulay S, Philip A, Solomon S. Influence of maternal diabetes on fetal rat development: Alteration of insulin receptors in fetal liver and lung. J. Endocrinol. 1983. 98(3):401410. DOI: 10.1677/joe.0.0980401.

35. Merzouk H, Madani S, Sari DC, Prost J, Bouchenak M, Belleville J. Time course of changes in serum glucose, insulin, lipids and tissue lipase activities in macrosomic offspring of rats with streptozotocin-induced diabetes. Clin Sci. 2000. 98(1):21-30. DOI: 10.1042/cs0980021.

36. Aerts L, Holemans K, Van Assche FA. Maternal diabetes during pregnancy: consequences for the offspring. Diabetes Metab Rev. 1990. 6(3): 147-67.

37. Holemans K, Aerts L, Van Assche FA. Evidence for an insulin resistance in the adult offspring of pregnant streptozotocin-diabetic rats. Diabetologia. 1991. 34(2):81-5.

38. Casanueva R, Viteri FE. Iron and oxidative stress in pregnancy. J Nutr. 2003. 133(5 Suppl 2):1700S-1708S.

39. Benzie IFF. Lipid peroxidation: a review of causes, consequences, measurements and dietary influences. Int J Food Sci Nutr. 1996. 47(3):233-61.

40. Huang J, Jones D, Luo B, Sanderson M, Soto J, Abel ED, Cooksey RC, McClain DA. Iron overload and diabetes risk: a shift from glucose to Fatty Acid oxidation and increased hepatic glucose production in a mouse model of hereditary hemochromatosis. Diabetes. 2011. 60(1):80-7. DOI: $10.2337 / \mathrm{db} 10-0593$.

41. Rajpathak SN, Crandall JP, Wylie-Rosett, J, Kabat GC, Rohan TE, Hu FB. The role of iron in type 2 diabetes in humans. Biochim Biophys Acta. 2009. 1790(7):671-81. DOI: 10.1016/j.bbagen.2008.04.005.

42. Yamano N, Ikeda Y, Sakama M, Izawa-Ishizawa Y, Kihira Y, Ishizawa K, Miyamoto L, Tomita S, Tsuchiya K, Tamaki T. A long-term high-fat diet changes iron distribution in the body, increasing iron accumulation specifically in the mouse spleen. J Nutr Sci Vitaminol (Tokyo). 2015. 61(1):20-7. DOI: $10.3177 /$ jnsv.61.20.

43. Fernández-Real JM, McClain D, Manco M. Mechanisms Linking Glucose Homeostasis and Iron Metabolism Toward the Onset and Progression of Type 2Diabetes. Diabetes Care. 2015. 38(11):2169-76. DOI: 10.2337/ dc14-3082.

44. Sangani RG, Ghio AJ. Iron, human growth, and the global epidemic of obesity. Nutrients. 2013. 5(10):4231-49. DOI:10.3390/nu5104231.

45. Leiva E, Mujica V, Sepúlveda P, Gusmán L, Núñez S, Or- 
rego R, Palomo I, Andrews M, Arredondo MA. High levels of iron status and oxidative stress in patients with metabolic syndrome. Biol Trace Elem Res. 2013. 151(1):1-8. DOI: $10.1007 / \mathrm{s} 12011-012-9525-3$.

46. Amiri FN, Basirat Z, Omidvar S, Sharbatdaran M, Tilaki $\mathrm{KH}$, Pouramir M. Comparison of the serum iron, ferritin levels and total iron-binding capacity between pregnant women with and without gestational diabetes. J Nat Sci Biol Med. 2013. 4(2):302-5. DOI: 10.4103/09769668.116977.

47. Fernández-Real JM, Moreno JM, López-Bermejo A, Chico B, Vendrell J, Ricart W. Circulating soluble transferrin receptor according to glucose tolerance status and insulin sensitivity. Diabetes Care. 2007. 30(3):604-8. DOI: 10.2337/dc06-1138.

48. Silva M, de Brito Magalhães CL, de Paula Oliveira R, Silva ME, Pedrosa ML. Differential expression of iron metabolism proteins in diabetic and diabetic iron-supplemented rat liver. J Biochem Mol Toxicol. 2012. 26(3): 123-9. DOI: 10.1002/jbt.20418.

49. Harrison PM, Arosio P. The ferritins: molecular properties, iron storage function and cellular regulation. Biochim Biophys Acta. 1996. 1275(3):161-203. DOI: 10.1016/0005-2728(96)00022-9.

50. Andrews NC. Iron metabolism: iron deficiency and iron overload. Annu Rev Genomics Hum Genet. 2000. 1:7598. DOI: 10.1146/annurev.genom.1.1.75.

51. Esposito BP, Breuer W, Sirankapracha P, Pootrakul P, Hershko C, Cabantchik ZI. Labile plasma iron in iron overload: Redox activity and susceptibility to chelation. Blood. 2003. 102(7):2670-7. DOI: http://dx.doi.org/10.1182/ blood-2003-03-0807.

52. Aljwaid H, White DL, Collard KJ, Moody AJ, Pinkney $\mathrm{JH}$. Non-transferrin-bound iron is associated with biomarkers of oxidative stress, inflammation and endothelial dysfunction in type 2 diabetes. J Diabetes Complications. 2015. 29(7):943-9. doi: 10.1016/j.jdiacomp.2015.05.017.

53. Davis RJ, Corvera S, Czech MP. Insulin stimulates cellular iron uptake and causes the redistribution of intracellular transferrin receptors to the plasma membrane. J Biol Chem. 1986. 261(19):8708-11.

54. Clairmont KB, Czech MP. Insulin injection increases the levels of serum receptors for transferrin and insulin-like growth factor-II/mannose-6-phosphate in intact rats. Endocrinology. 1990. 127(4):1568-73. DOI: http://dx.doi. org/10.1210/endo-127-4-1568.

55. Ramos-Alves FE, de Queiroz DB, Santos-Rocha J, Duarte GP, Xavier FE. Effect of age and cyclooxygenase-2-derived prostanoids on the progression of adult vascular dysfunction in offspring diabetic rats. $\mathrm{Br} \mathrm{J}$
Pharmacol. 2012. 166(7):2198-2208. DOI: 10.1111/j. 1476-5381.2012.01945.x.

56. Zafar U, Qureshi HJ, Imran M. Comparison of iron status and insulin resistance between non-diabetic offsprings of type 2 diabetics and non-diabetics. J Ayub Med Coll Abbottabad. 2015. 27(2):307-11.

57. Lewis RM, Petry CJ, Ozanne SE, Hales CN. Effects of maternal iron restriction in the rat on blood pressure, glucose tolerance, and serum lipids in the 3-month-old offspring. Metabolism. 2001. 50(5):562-7.

58. Aso Y et al. Relation between serum high molecular weight adiponectin and serum ferritin or prohepcidin in patients with type 2 diabetes. Diabetes Res Clin Pract. 2010. 90(3):250-5. DOI: 10.1016/j.diabres.2010.09.008.

59. Fujita $\mathrm{N}$ et al. Hepcidin Expression in the Liver: Relatively Low Level in Patients with Chronic Hepatitis C. Mol Med. 2007. 13(1-2):97-104. DOI: 10.2119/2006-00057. Fujita

60. Torti FM, Torti SV. Regulation of ferritin genes and protein. Blood. 2002. 99(10):3505-3516.

61. Fairbanks VG, Beutler E. Iron metabolism. In: Beutler E, Lichtman MA, Coller BS, Kipps TJ, Seligsohn U. Williams-Hematology. New York: Mcgraw-Hill; 2001.p. 295-304.

62. Lima E, Parra D. Peroxidação lipídica: mecanismos e avaliação em amostras biológicas. Rev Bras Cienc Farm. 2001. 37(3): 1-23

63. Bonnes-Taourel D, Guérin MC, Torreilles J. Is malondehyde a valuable of peroxidation? Biochem Pharmacol. 1992. 44(5):985-8. DOI:10.1016/0006-2952(92)90132-3.

64. Cockell KA, Wotherspoon AT, Belonje B, Fritz ME, Madère R, Hidíroglou N, Plouffe LJ, Ratnayake WM, Kubow S. Limited effects of combined dietary copper deficiency/ iron overload on oxidative stress parameters in rat liver and plasma. J. Nutr Biochim. 2005. 16(12):750-6.

65. Kinalski M, Sledziewski A, Telejko B, Zarzycki W, Kinalska I. Lipid peroxidation and scavenging enzyme activity in streptozotocin-induced diabetes. Acta Diabetol. 2000. 37(4):179-83.

66. Kosower NS, Kosower EM. The glutathione status of cells. Int Rev In cytol. 1978. 54:109-60.

67. Meister A, Anderson ME. Glutathione. Annu Rev Biochem. 1983. 52:711-60. DOI: 10.1146/annurev. bi.52.070183.003431.

68. Galleano M, Puntarulo S. Role of antioxidants on the erythrocytes resistence to lipid peroxidation after acute iron overload in rats. Biochim Biophys Acta. 1995, 1271(23):321-6. DOI:10.1016/0925-4439(95)00049-A.

69. Deneke SM, Fanburg BL. Regulation of cellular glutathione. Am J Physiol. 1989. 257(4 Pt 1):L163- 